\title{
INTERACTIVE MODELLER FOR EQUIPMENT OPERATION IN LONG-SPAN BRIDGE CONSTRUCTION
}

\author{
Byungil Kim \\ ikim@yonse.ac.kr \\ Changyoon Kim \\ changyoonkim@yonsei.ac.kr \\ Wonil Lee \\ leaveatrail@naver.com \\ Hyoungkwan Kim \\ hyoungkwan@yonsei.ac.kr \\ Yonsei University, Seoul, Korea
}

\begin{abstract}
Three dimensional (3D) animation of construction simulation makes communication among different project participants effective by visualizing the construction operation. The effect of 3D animation becomes stronger in a more complex operation. However, the modelling process of the 3D animation requires time-consuming efforts. Thus, interactive modelling environment is strongly preferred where equipment, materials, and temporary structures can easily and freely be placed by the construction engineers in a real-time manner. This research aims to develop an equipment operation modeller that can allow users to interactively communicate with the system for long-span bridges. Augmented reality is used as the main methodology for implementing the modeller. The proposed modeller is expected to significantly improve the planning process of construction equipment operations.
\end{abstract}

KEYWORDS: Cable-stayed bridge construction; Construction equipment; Computer simulation; 4D CAD; Augmented reality

\section{INTRODUCTION}

Three or four dimensional (3D or 4D) CAD models have been developed to improve the understanding of construction spatial constraints. Those models have enhanced not only the planning process but also the construction process by being able to predict potential problems of construction sites. Recently, one of the main focuses in the 3D/4D research community has been placed on a more detailed level of constructability analysis than those of earlier 3D/4D studies. In other words, they started to focus on the efficiency and safety issues of construction equipment operation than activity level management (Al-Hussein et al. 2006; Park et al. 2009).

The operational level 3D/4D CAD models are in many aspects different from those of activity level models that mainly show only finished or unfinished static status of certain components of the facility. The operation level models are more detailed versions of activity 
level models, such that the operation models deal with the description of actual movement of construction equipment and locations of material. The operation level models require more intuitive and interactive communication between the users and the computer system. For example, in case of a derrick crane operation in a cable-stayed bridge, the crane's location and its working envelope has to be carefully analyzed with respect to the spatially constrained environment of the long-span bridge construction site. Thus, it is desirable to use a system that intrinsically provides an intuitive, interactive platform where users can freely test various operation scenarios of construction equipment.

This paper proposes a new system that allow the users to interactively test various scenarios of construction equipment operation. The system relies on the concept of 3D/4D CAD and augmented reality technology. An experimental prototype is presented for validating the technical feasibility and potential benefit of the proposed system in the case of a derrick crane operation of a cable-stayed bridge construction.

\section{LITERATURE REVIEW}

Efforts have been made to effectively develop and apply augmented reality (AR) to construction. Webster et al. (1996) developed a pioneering AR system to improve methods for visualization and inspection of architectural structures. Dunston and Wang (2005) proposed the development of AR-based computer interface and studied human factors issues with AR models. Kamat and El-Tawil (2007) evaluated AR for rapid assessment of building damage induced by earthquake. Shin and Dunston (2009) presented the development of an AR prototype system for steel column inspection.

Milgram and Colquhoun (1999) presented the reality-virtuality continuum to explain how to classify the AR systems. The continuum provided a means to perceive AR studies in terms of the level of virtuality (or reality) and viewpoints of the users.

According to the classification method, previous research efforts have been made mainly for construction workers or operators' viewpoints. In case of crane operation, the operator sitting in the cab of the crane was represented in the AR studies. However, for effective understanding of the whole construction site information related to spatial constraints, the viewpoint from the above is also required. That is, the perspective of somebody with global awareness is necessary to make sure the equipment does not collide with its surrounding work environment. This lack of studies in the particular research domain is the main motivation behind our research to develop a AR-based system that can provide a world view to interactively test the operation of construction equipment.

\section{SYSTEM ARCHITECTURE}

The main purpose of the proposed system is to be able to interactively test various construction scenarios containing movement of materials and equipment. However, the benefit of the system can be significantly increased with additional functionalities such as cost estimation models because the interactive system can be used for more efficient construction management purposes. Figure 1 shows the main components of the proposed system. 
(1) ESTIMATION MODELS

- Cost model

- Time model

- Environmental burden model

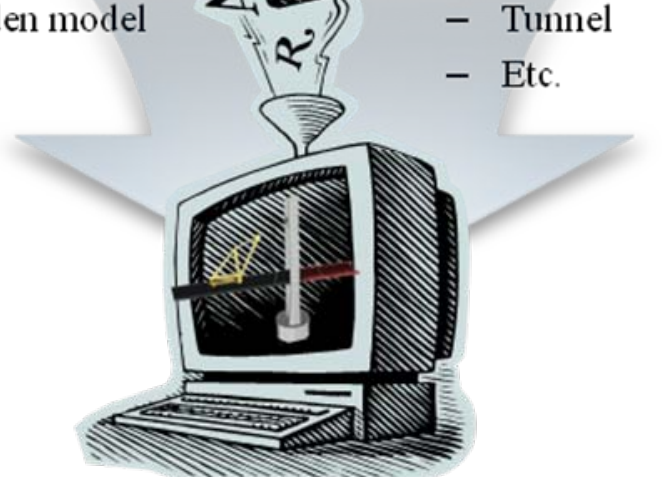

(3) INTERACTIVE MODELLER

Figure 1: The three main components of the proposed system

The system is comprised of three main components: estimation models, a 3D model authoring tool, and an interactive modeller. The estimation models are for the users to easily access such information as cost, time and environmental burden information. For instance, if the users want to know the fabrication cost and installation schedule of a precast concrete panel, they can pinpoint the panel on the screen using mouse or other devices. Once the objects are selected, its related information will pop out on the screen. In another example, when a derrick crane is selected, the trade-off issue of its operation efficiency and estimated cost can be considered so that the users can choose the equipment best suited for the particular construction site.

The 3D model authoring tool is another main component of the proposed system. The particular type of construction project will determine the way the 3D models are easily generated. That is, different modelling methods will be required for a range of construction projects such as tunnels, industrial plants, and large-scale earth works. Even different size and shape of construction equipment should be designed using this authoring tool unless otherwise provided. Parametric modelling will be the major methodology for implementing the authoring tool.

The interactive modeller allows for the integrated environment where the management information from the estimation models and the 3D size and shape information from the authoring tool are merged. Figure 2 shows how the proposed system will be used for a construction project. At the field office, multiple project participants can have a collaborative meeting to discuss the most suitable solution for a construction operation. The prime contractor, subcontractors and consultants can be gathered to express their opinions in an intuitive way, thanks to the increased reality of the AR-based modeller. In addition, the ongoing discussions can be monitored by the personnel who are remotely located at the contractor's home office. 


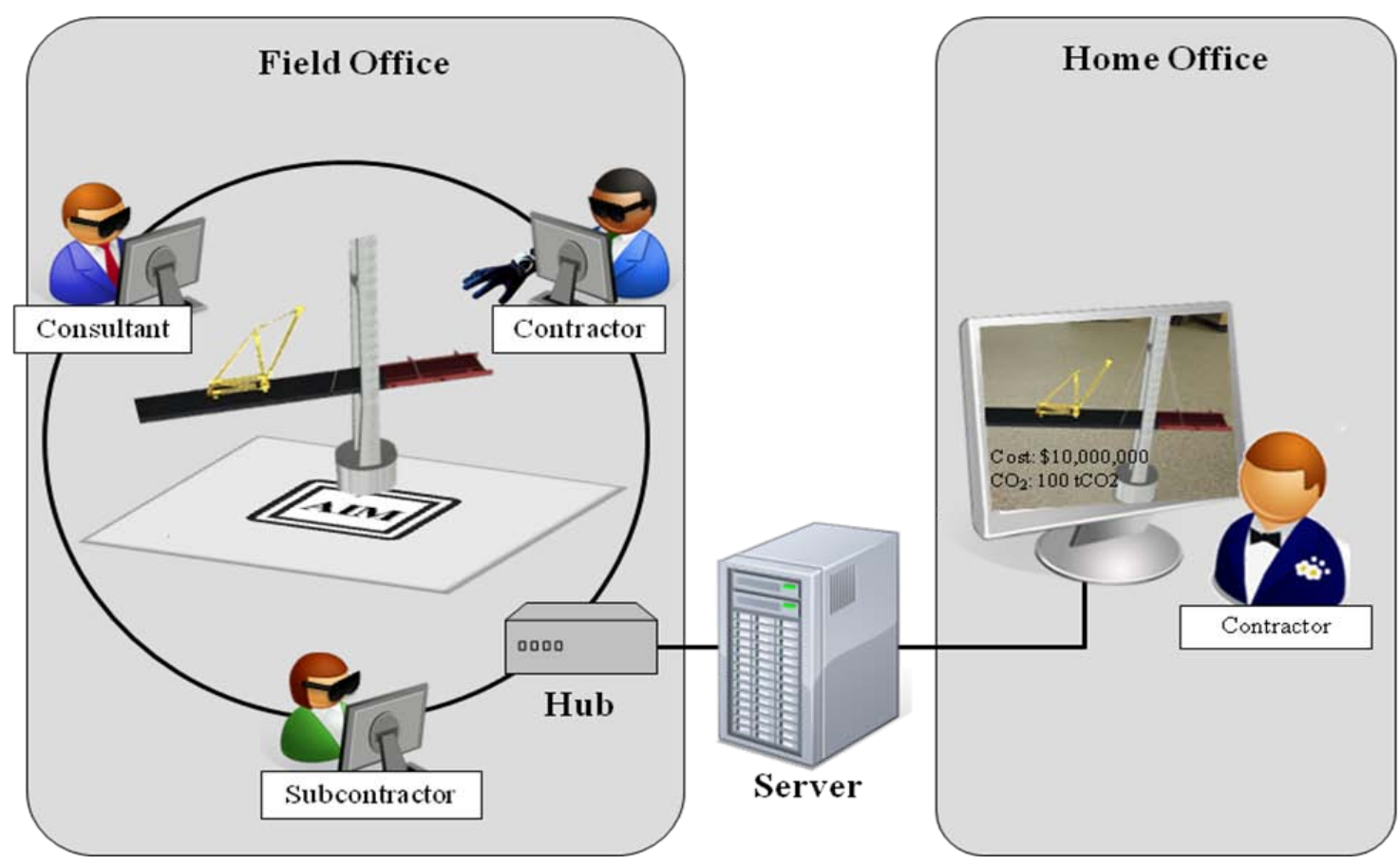

Figure 2: Usage of the proposed system

\section{EXPERIMENTAL PROTOTYPE FOR BRIDGE CONSTRUCTION}

An experimental prototype was developed with an emphasis on the interactive modeller of the proposed system. As for now, the estimation models are not yet developed and AutoCAD Revit Architecture and 3ds Max played the role of the 3D model authoring tool. The Revit Architecture was used to create the 3D models of the equipment and the structure, as detailed in Park et al. (2009). The model was then imported to the 3ds Max environment, to be exported to the VRML (Virtual Reality Modelling Language) format. The VRML model was then able to be used in the ARToolkit environment.

Figure 3 shows the derrick crane model imported to the 3ds Max environment. In the Max environment, a rendering process was conducted to have a painting effect on the equipment. Figure 4 shows the augmented realty of the derrick crane shown above the marker. In the same manner, bridge upper decks and pylons were converted to the VRML format and displayed on top of its corresponding marker. Figure 5 shows the AR image of the derrick crane placed on the bridge. The derrick crane and bridge each had one corresponding marker and the location of the virtual image of derrick crane with respect to the marker center had to be adjusted so as to be placed on top of the bridge deck.

Figure 6 shows the collaborative platform in which multiple participants can express their ideas as to how the construction equipment operation should be done. The common medium of communication in this case becomes the markers on the table. If a user wants to move or rotate the derrick crane, they can physically move or rotate the corresponding marker. Then, the changed status of the construction scenario is also reflected on the image being watched by the other user. In this way, they can test different locations or scenarios of construction operation, which are shared by the system users in real time. 


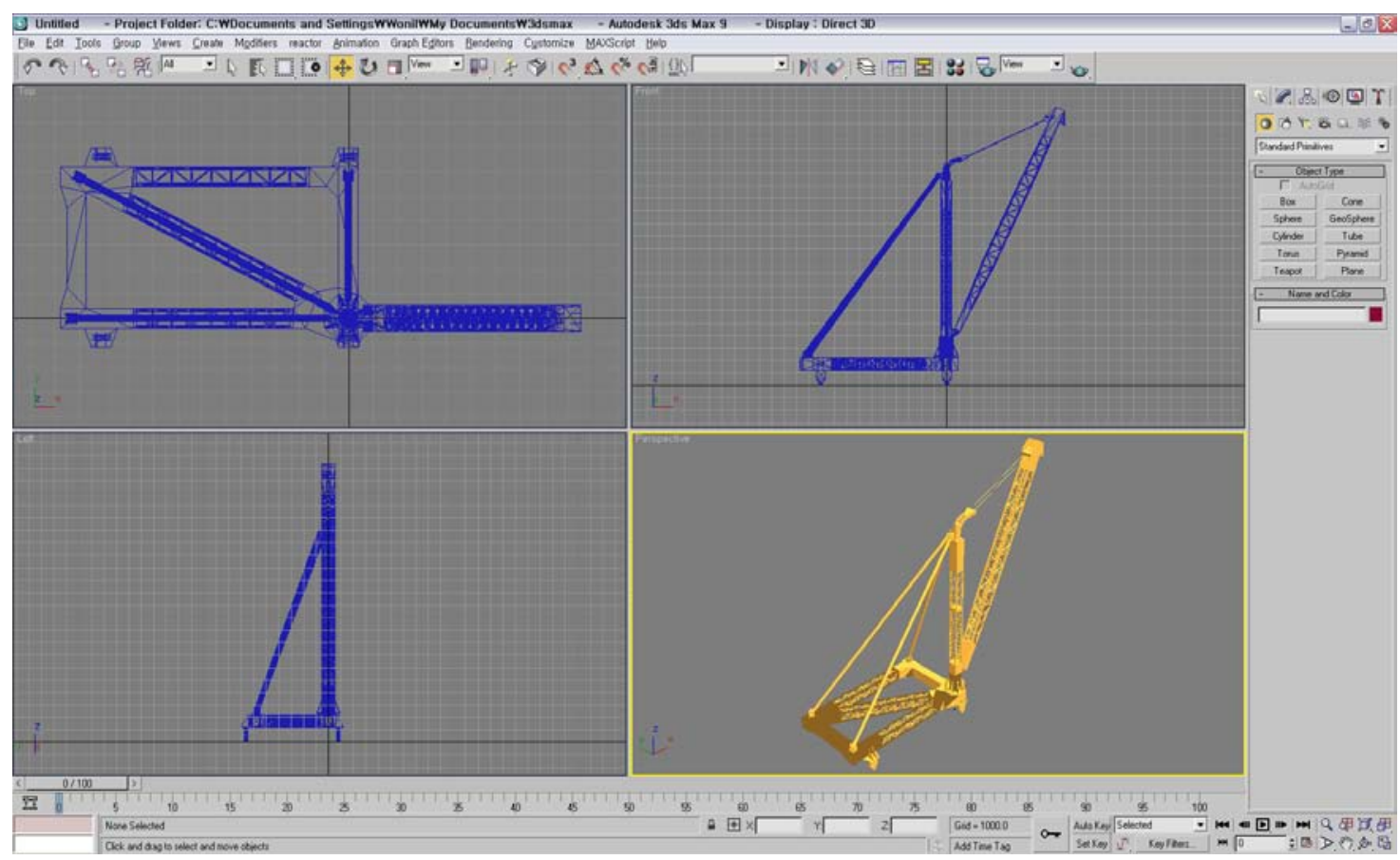

Figure 3: Derrick crane model conversion to the 3ds Max format

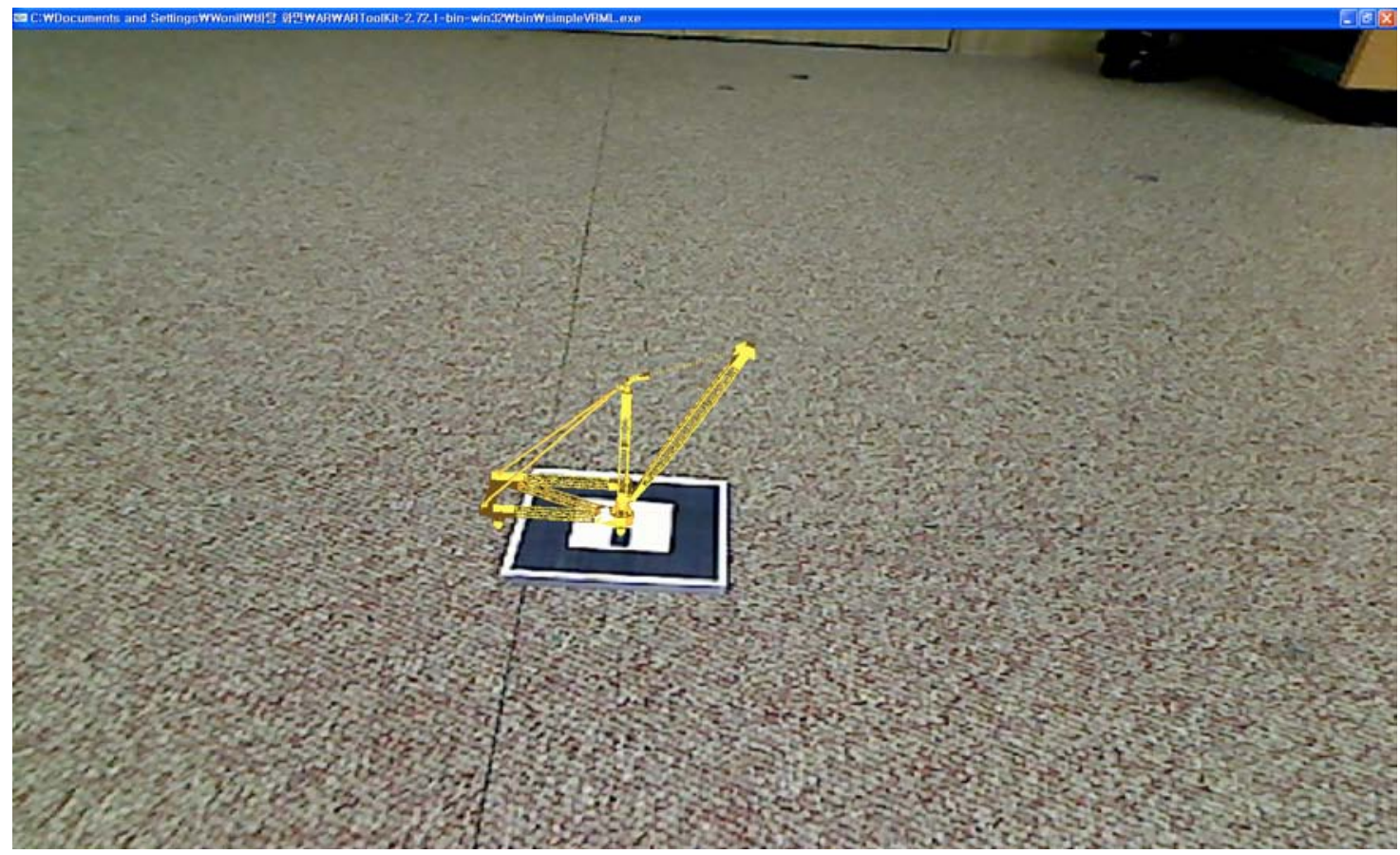

Figure 4: Derrick crane displayed on the mark image 


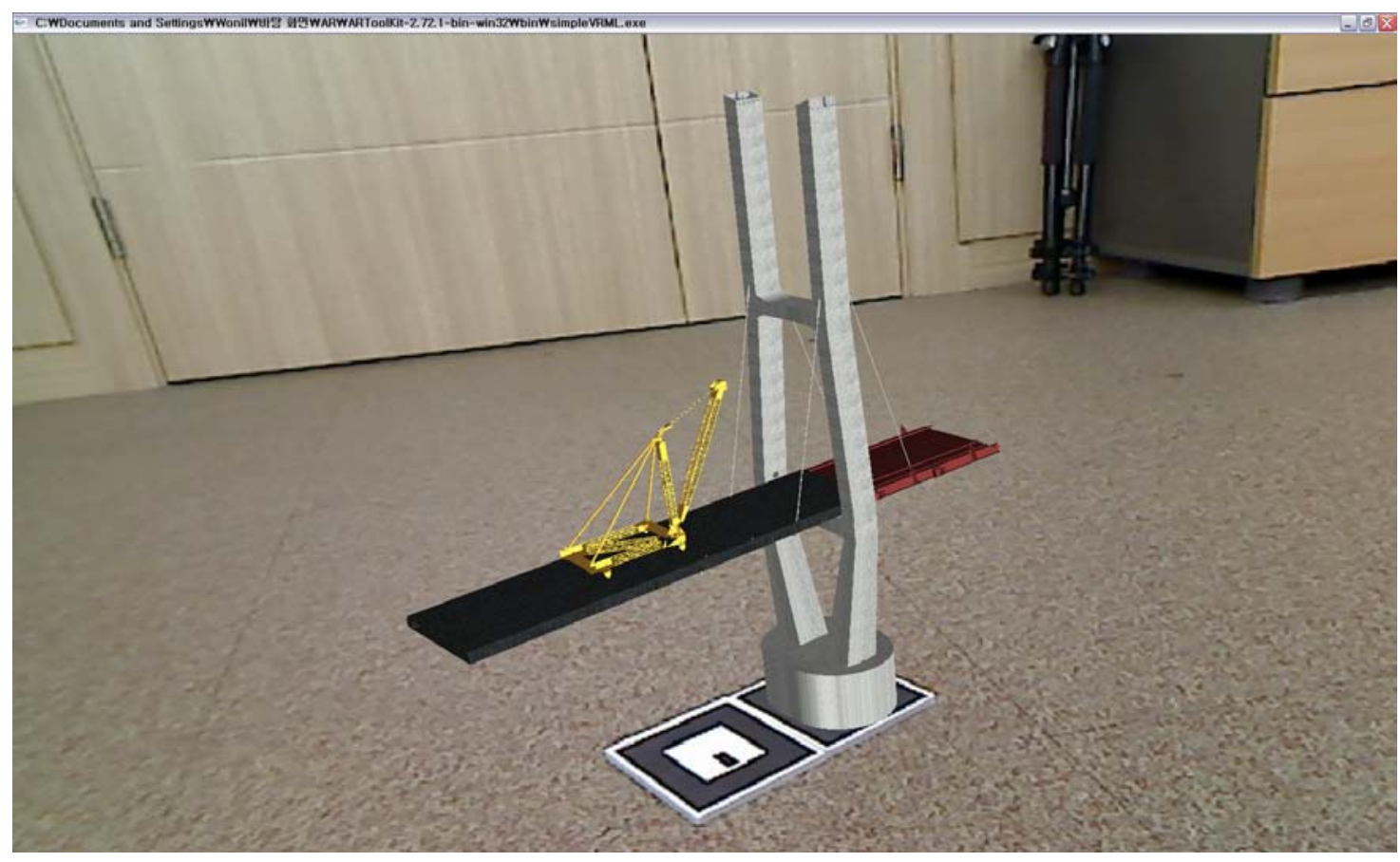

Figure 5: Derrick crane placed on the cable-stayed bridge

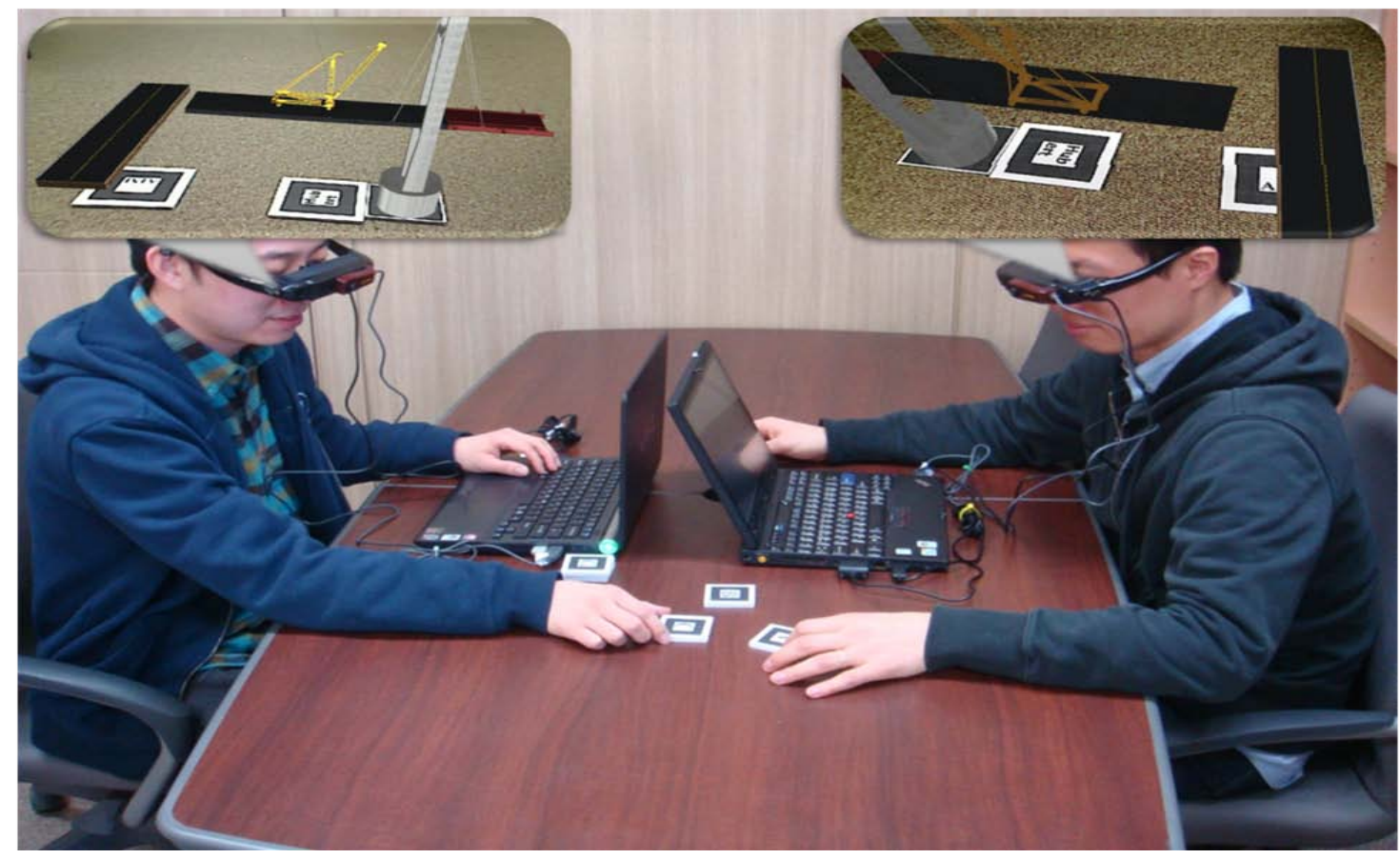

Figure 6: Collaborative platform for the derrick crane operation 


\section{CONCLUSIONS AND RECOMMENDATIONS}

This paper proposed an interactive system that allows the users to collaboratively test various construction operation scenarios. The system was comprised of the three main components: estimation models, a 3D model authoring tool, and an interactive modeller. The interactive modeller, with the help of augmented reality, is able to combine the construction management information delivered by the estimation models and the 3D shape information from the 3D model authoring tool. An experimental prototype was also presented for the derrick crane operation in a bridge construction. The prototype validated the technical feasibility and potential benefit of the proposed system.

Future studies are required for the full implementation of the proposed system. The estimation models can incorporate diverse management issues into the system. In particular, the information related to environmental friendliness in a construction site can accompany the augmented reality model. For instance, each 3D component's environmental impact such as greenhouse gas emission amount can be attached to the AR model, which will make it easier to monitor the environmental aspect of the construction project. The proposed system, once completed, is expected to become an interactive, collaborative planning tool for improved construction operations.

\section{ACKNOWLEDGEMENTS}

This work was supported by National Research Foundation of Korea Grant funded by the Korean Government (2009-0075985).

\section{REFERENCES}

Al-Hussein, M., Niaz, M. A., Yu, Haitao, and Kim, H. (2006) Integrating 3D visualization and simulation for tower crane operations on construction sites. Automation in Construction, 15(5), 554-562.

Dunston, P., Shin, D. H. (2009) Technology development needs for advancing Augmented Reality-based inspection. Automation in Construction, in press.

Dunston, P., Wang, X. (2005) Mixed reality-based visualization interfaces for the architecture, engineering, and construction industry. Journal of Construction Engineering and Management, 131(12), 1301-1309.

Kamat, V. R., El-Tawil, S. (2007) Evaluation of augmented reality for rapid assessment of earthquake-induced building damage. Journal of computing in civil engineering, 21(5), 303310.

Milgram, P., and Colquhoun, H. Jr. (1999) A taxonomy of real and virtual world display integration. Mixed reality: Merging real and virtual worlds, Y. Ohta and H. Tamura, eds., Ohmsha Ltd. And Springer, New York, 5-30. 
Park, T., Kim, M. K., Kim, C., and Kim, H. (2009) Interactive 3D CAD for effective derrick crane operation in a cable-stayed bridge construction. Journal of Construction Engineering and Management, 135(11), 1261-1274.

Webster, A., Feiner, S., MacIntyre, B., Massie, W., and Krueger, T. (1996) Augmented reality in architectural construction, inspection, and renovation. Proc., ASCE Computing in Civil Engineering, J. Vanegas and Paul Chinowsky, eds., ASCE, Reston, Va., 913-919. 